\title{
Research article \\ A comparative study on effect of platelet rich plasma Vs conventional dressing in healing rate of chronic diabetic ulcers
}

\author{
Swathika Rajendran ${ }^{1}$, Manuneethimaran Thiyagarajan ${ }^{2}$, Balaji Singh K. ${ }^{3}$, Nitesh Navrathan ${ }^{4}$ \\ ${ }^{1}$ Post Graduate, ${ }^{2}$ Associate Professor, ${ }^{3}$ Professor, ${ }^{4}$ Assistsnt Professor, General Surgery Department, Sri Ramachandra Medical \\ University, Porur, Chennai, Tamil Nadu, India
}

(Received: November $2020 \quad$ Revised: October $2021 \quad$ Accepted: November 2021)

Corresponding author: Nitesh Navrathan.Email: niteshachha87@gmail.com

\begin{abstract}
Introduction and Aim: This study has been undertaken to evaluate efficacy of platelet rich plasma (PRP) dressing that is recently introduced with conventional dressing. We have tried to compare PRP dressing with conventional normal dressing in healing the wound.

Materials and Methods: We conducted this study in 120 patients of chronic non healing diabetic foot ulcers who were treated over the period of three years. Number of cases (60 each) were studied for efficacy of applying autologous platelet rich plasma versus normal dressing for chronic non healing diabetic foot ulcers. At every week follow up, the ulcer was examined for granulation tissue, area and volume which was calculated using Vernier calipers and marked on a graph paper and photographs were taken. Wound healing was compared between PRP and normal dressing.

Results: The incidence of male susceptibility was more (70\%) than females (30\%) in diabetic foot. Average rate of healing per week was found to be significantly better among the PRP group than normal dressing group. The ulcer showed significant reduction in size among the PRP group in comparison to the normal dressing group with a significant $\mathrm{P}$ value (0.001).

Conclusion: In this study application of platelet rich plasma into chronic non-healing diabetic foot ulcers in comparison with conventional dressing has shown a significant reduction in the size of the ulcer, hospital stay and cost for the patient.
\end{abstract}

Keywords: Diabetic ulcer; platelet rich plasma therapy; diabetic foot; wound healing; chronic diabetic wound healing; non-healing ulcer.

\section{INTRODUCTION}

$\mathrm{D}$ efinition of non-healing wound is chronic wound which fails to heal within 3 months (1). $0.1 \%$ of western population is suffering from non-healing ulcers. Venous leg ulcers, pressure ulcers, ischemic wounds and diabetic foot ulcers are common chronic ulcers (2). In western countries total cost of $2-4 \%$ of health budgets used for chronic wound managements (3). Wound healing is included with stages of inflammation, proliferation, repair, and remodeling $(4,5)$. In the part of platelet degranulation is, the inflammatory stage of healing is started (4).

platelet-derived growth factor (PDGF), platelet factor 4 , transforming growth factor are included in Growth factors $-\mathrm{p}$, platelet-derived angiogenesis factor and platelet derived epidermal growth factor. All these five factors help in tissue formation and epithelization of wounds $(4,6)$. On their release, these factors stimulate and modulate multiple biological processes that are important in wound healing (4-7). In some cases, lack growth factors occur in Chronic wounds. Decreased production, decreased release, trapping, excess degradation, or a combination of these mechanisms may be the cause of Decreased growth factor availability (5).
Recent studies have found that accelerate wound healing might be caused by platelet-rich plasma (PRP) $(8,9)$. Margolis et al., (9) Showed that $>6000$ patients increased wound healing with PRP, with the effect being best in those with the most severe wounds. An indication is there which shows PRP has infection-fighting properties. The role in recruiting white blood cells (10) and releasing bactericidal factors and are played by platelets (11). PRP use in chronic wounds are made more attractive by revelations because they are plagued by infection. Contemporary management of chronic wounds improve natural healing (12). There is no study to find out the efficacy of PRP in wound healing. In this study we are trying to show the efficacy of PRP dressing.

The aim of this study was to evaluate the wound healing efficacy of applying autologous platelet rich plasma into chronic non healing diabetic foot ulcers versus normal dressing.

\section{MATERIALS AND METHODS}

Present study was carried out at Sri Ramachandra University, where 120 patients of chronic non healing diabetic foot ulcers were treated over the period of three years (2017-2019). Consent was obtained for 
each patient before including into the study. Patients were randomized into two groups by simple randomization by using flip card. we have made two groups of patients with 60 patients in each group. one group PRP dressing was applied, and another group normal dressing was applied.

\section{Inclusion criteria}

Patients with chronic non healing diabetic foot ulcer of duration more than 6 weeks

Diabetic patients with $\mathrm{HbA1c}$ of $<9$

Size of the ulcer between $5-10 \mathrm{cms}$

Wagner's class 2 ulcers (13)

\section{Exclusion criteria}

Patients with a bleeding disorder

Uncontrolled sugar levels of $\mathrm{HbAlc}>9$

Diabetic nephropathy

Associated venous (or) arterial disease

Anemia

> Wagner's class 2 ulcers and size > $10 \mathrm{~cm}$ (13)

The ulcer size (length, breadth, and depth) was measured with Vernier calipers before starting the treatment.

\section{PRP preparation}

First, $20 \mathrm{ml}$ of venous blood was withdrawn, then, we have it added to a test tube containing acid citrate dextrose in a ratio of 9:1 (blood: acid citrate dextrose). This solution was centrifuged at $5000 \mathrm{rpm}$ for $15 \mathrm{~min}$ to separate the red blood cells from the platelets and plasma. After that, we have collected the supernatant and the buffy coat, which composed of platelets and plasma. This solution centrifuged again at $2000 \mathrm{rpm}$ for $5-10 \mathrm{~min} .1 .5 \mathrm{ml}$ of the bottom layer was taken and $10 \%$ calcium chloride was added $(0.3 \mathrm{ml}$ for $1 \mathrm{ml}$ of PRP). The mean vale of the platelet count was 3.5 lakhs/mm (SD 0.95) and the mean value of final concentration of platelets in PRP was 6.25 Lakhs/mms (SD 1.25).

Thus, the activated PRP was applied into the wound after proper surgical debridement and dressed with a non-absorbent dressing. Culture swab from the wound was obtained and appropriate antibiotics were started according to sensitivity report. This process was repeated once weekly for 6 weeks. At every week follow up, the ulcer was examined for granulation tissue, area and volume which was calculated using Vernier calipers and marked on a graph paper and photographs were taken.

Wound area was calculated using the formula for an ellipse: Length x width x o 7854 (an ellipse is closer to a wound shape than a square or rectangle that would be described by simple length $\mathrm{x}$ width). The use of an ellipse for calculating wound measurement has been used in RCTs in wound healing literature $(11,13)$. Volume was calculated using the formula length $\mathrm{x}$ width $\mathrm{x} 0.7854) \mathrm{x}$ depth (100).

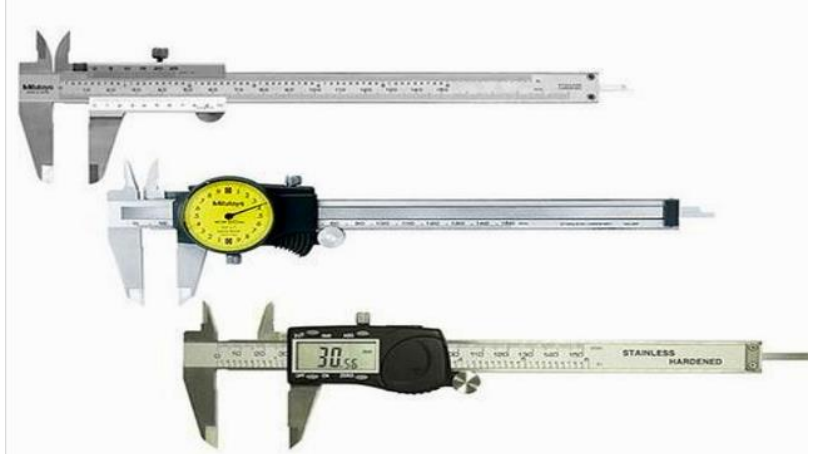

Fig. 1: Vernier caliper used to measure the wound

The treatment outcome was defined as a percentage change of the volume, which will be calculated as initial measurement minus assessment day measurement divided by initial measurement. Area of the wound was measured at regular intervals every week using length and width of the ulcer. Same procedure was done for ulcers in both group of patients. The wounds that showed sufficient granulation but failed to re-epithelize will be grafted with autologous split skin graft in both PRP and NonPRP group. Wounds that showed partial granulation or no granulation or increase in size of ulcer during the treatment period will be classified under nonhealing ulcers. Wound size reduction was compared in both groups and results were tabulated in software analysis.

\section{Statistical analysis}

To describe about the data descriptive statistics frequency analysis, percentage analysis was used for categorical variables and the mean \& S.D were used for continuous variables. To find the significant difference between the bivariate samples in independent groups the unpaired sample t-test was used. For the multivariate analysis in repeated measures the repeated measures of ANOVA with adjustment for multiple comparisons to control the type I error, the Bonferroni test was used. Chi-Square test was used to find the significance in categorical data. In all the above statistical tools the probability value 0.05 is considered as significant level.

\section{RESULTS}

Table 1: Age in diabetic foot

\begin{tabular}{|c|c|c|c|}
\hline $\begin{array}{c}\text { Age in } \\
\text { years }\end{array}$ & PRP & ND & $\begin{array}{c}\text { Age distribution } \\
\text { in the study }\end{array}$ \\
\hline $21-30$ & 02 & 02 & $4(3.3 \%)$ \\
\hline $31-40$ & 00 & 10 & $10(8.3)$ \\
\hline $41-50$ & 24 & 16 & $40(33.3 \%)$ \\
\hline $51-60$ & 22 & 18 & $40(33.3 \%)$ \\
\hline $61-70$ & 12 & 10 & $22(18 \%)$ \\
\hline $71-80$ & 00 & 04 & $4(3.3 \%)$ \\
\hline Total & 60 & 30 & 120 \\
\hline
\end{tabular}

It is seen from this table that diabetic foot ulcers are more seen in $4^{\text {th }}-5^{\text {th }}$ decade and $5^{\text {th }}$ to $6^{\text {th }}$ decade 
(33.3\% each). The incidence of male susceptibility was more 82 patients $(68.3 \%)$ than females 82 $(31.7 \%)$ in diabetic foot.

Most of the patients had good glycemic control with (Hb A1c 7.6 to 8.5.). The mean hbA1 c in PRP dressing 7.83 and normal dressing 8.22.
In this study the most common organism isolated was Staphylococcus comprising $40(33 \%)$ patients and least common organism was E. coli and Proteus comprising of $4(3.3 \%)$ patients each and klebsiella $8(6.7 \%)$. Sterile cultures were found in $32(26.6 \%)$ patients.

Table 2: Average rate of healing per week

\begin{tabular}{|c|c|c|c|c|}
\hline \multicolumn{2}{|c|}{ Groups } & $\mathrm{N}$ & $\begin{array}{c}\text { Mean } \\
\left(\mathrm{cm}^{2}\right)\end{array}$ & $\begin{array}{c}\text { Standard } \\
\text { deviation }\end{array}$ \\
\hline \multirow{2}{*}{0 Weeks } & Normal dressing & 60 & 40.07 & 18.58 \\
\cline { 2 - 5 } & PRP dressing & 60 & 42.90 & 19.39 \\
\hline \multirow{2}{*}{2 Weeks } & Normal dressing & 54 & 38.56 & 18.53 \\
\cline { 2 - 5 } & PRP dressing & 60 & 31.54 & 13.45 \\
\hline \multirow{2}{*}{4 Weeks } & Normal dressing & 52 & 37.00 & 17.82 \\
\cline { 2 - 5 } & PRP dressing & 60 & 21.54 & 11.56 \\
\hline \multirow{2}{*}{6 Weeks } & Normal dressing & 52 & 35.39 & 17.08 \\
\cline { 2 - 5 } & PRP dressing & 60 & 9.94 & 9.25 \\
\hline \multirow{2}{*}{ Average healing } & Normal dressing & 52 & 0.83 & 0.78 \\
\cline { 2 - 5 } & PRP dressing & 60 & 5.49 & 3.27 \\
\hline
\end{tabular}

Table 2 shows, the wound size reduction in normal dressing group is mean wound size of $40.07 \mathrm{sq} . \mathrm{cm}$ in 0 week to 35.39 sq.cm in $6^{\text {th }}$ week. On the other hand, in PRP dressing group mean wound size of 42.9 sq. $\mathrm{cm}$ in 0 week to 9.94 sq.cm in $6^{\text {th }}$ week.
Average rate of healing per week was found to be 0.83 sq. cm in normal dressing and 5.49 sq.cm in PRP dressing group. This is statistically significant $(\mathrm{p}<0.001)$.

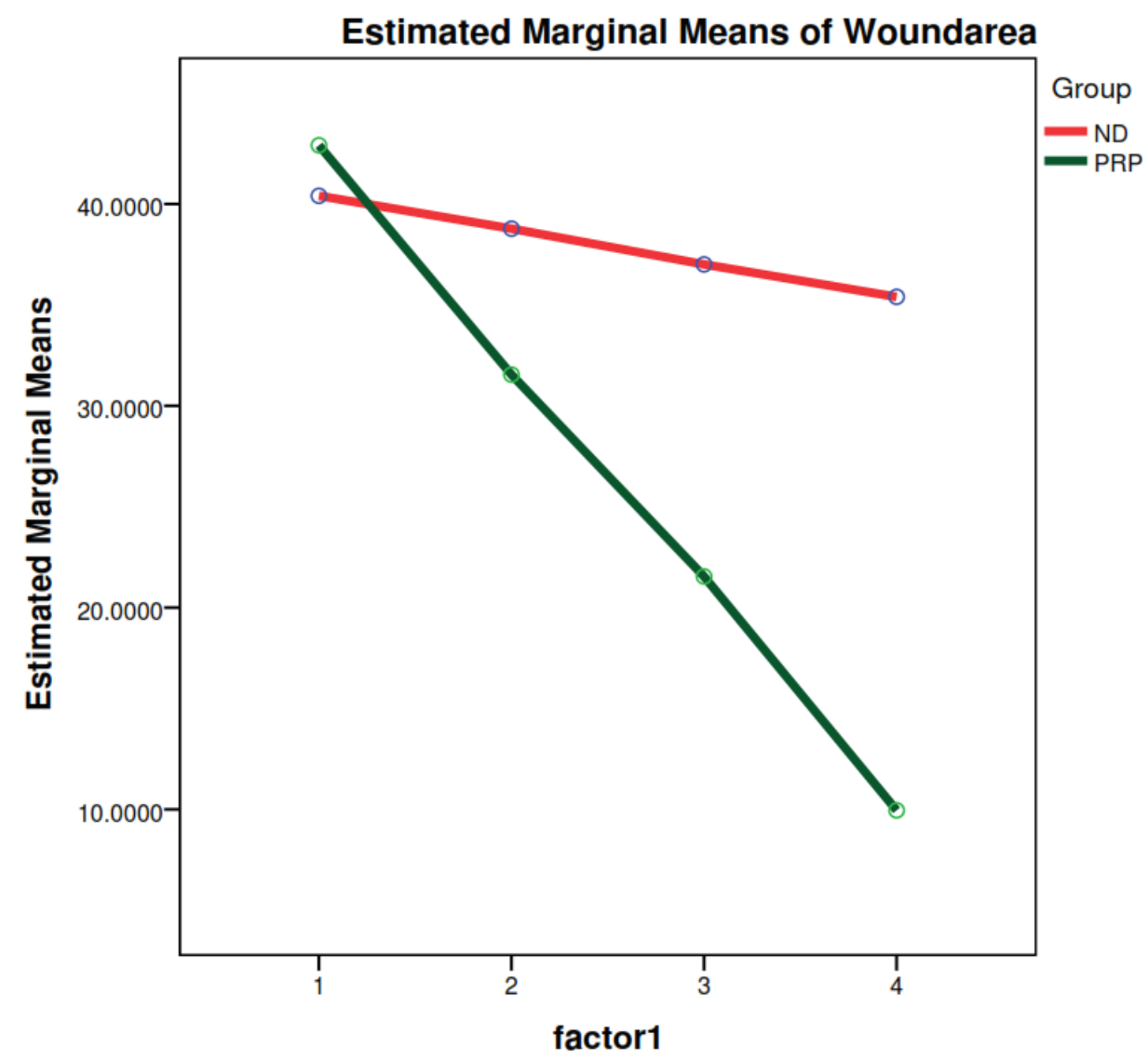

Chart 1: Mean reduction of wound area among both groups

The ulcer showed significant reduction in size among the PRP group in comparison to the normal dressing group with a significant $\mathrm{P}$ value (0.001). 
Table 3: Fate of the ulcer

\begin{tabular}{|c|c|c|c|c|}
\hline & \multicolumn{2}{|c|}{ Groups } & \multirow[b]{2}{*}{ Total } \\
\hline & & $\begin{array}{l}\text { Normal } \\
\text { dressing }\end{array}$ & $\begin{array}{c}\text { PRP } \\
\text { dressing }\end{array}$ & \\
\hline \multirow{2}{*}{ Full granulation } & Count & 2 & 6 & 8 \\
\hline & $\%$ within Groups & $3.3 \%$ & $10.0 \%$ & $6.7 \%$ \\
\hline \multirow{2}{*}{ Healed } & Count & 0 & 40 & 40 \\
\hline & $\%$ within Groups & $0.0 \%$ & $66.7 \%$ & $33.3 \%$ \\
\hline \multirow{2}{*}{ Non healing } & Count & 20 & 0 & 20 \\
\hline & $\%$ within Groups & $33.3 \%$ & $0.0 \%$ & $16.7 \%$ \\
\hline \multirow{2}{*}{ Partial granulation } & Count & 22 & 6 & 28 \\
\hline & $\%$ within Groups & $36.7 \%$ & $10.0 \%$ & $23.3 \%$ \\
\hline \multirow{2}{*}{ SSG } & Count & 8 & 8 & 16 \\
\hline & $\%$ within Groups & $13.3 \%$ & $13.3 \%$ & $13.3 \%$ \\
\hline \multirow{2}{*}{ Amputation } & Count & 8 & 0 & 8 \\
\hline & $\%$ within Groups & $13.3 \%$ & $0.0 \%$ & $6.7 \%$ \\
\hline \multirow{2}{*}{ Total } & Count & 60 & 60 & 120 \\
\hline & $\%$ within Groups & $100.0 \%$ & $100.0 \%$ & $100.0 \%$ \\
\hline
\end{tabular}

In Table 3, complete healing was identified in 40 patients $(66.7 \%)$ in PRP group but 0 patient in normal dressing group. There are 20 patients (33.3\%) in normal dressing group shows non healing wound but in PRP dressing it is 0\%. Split skin grafting was done in 8 patients $(13.3 \%)$ in each group. There were 8 patients (13.3\%) among the normal dressing group underwent amputation during the course due to gangrene and no patient underwent amputation in PRP group.

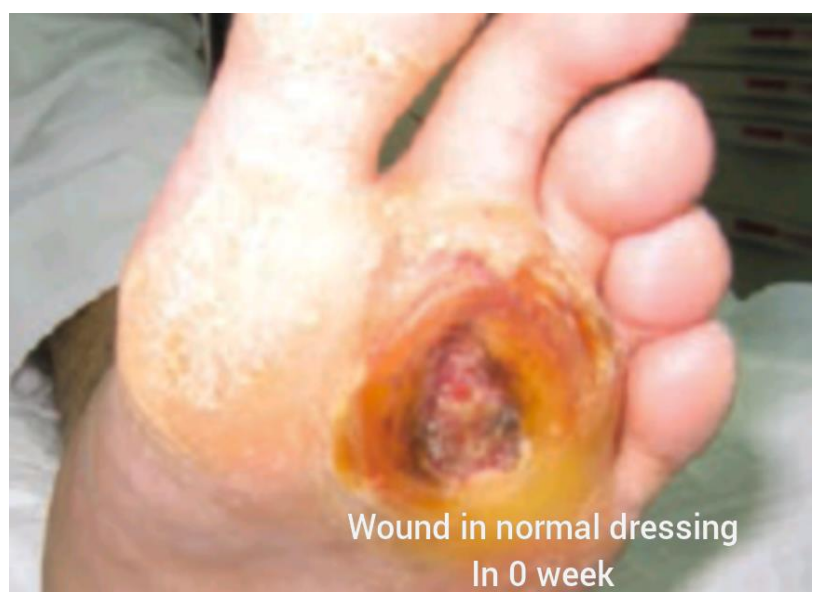

Fig. 2: Wound in 0 week (normal dressing group)

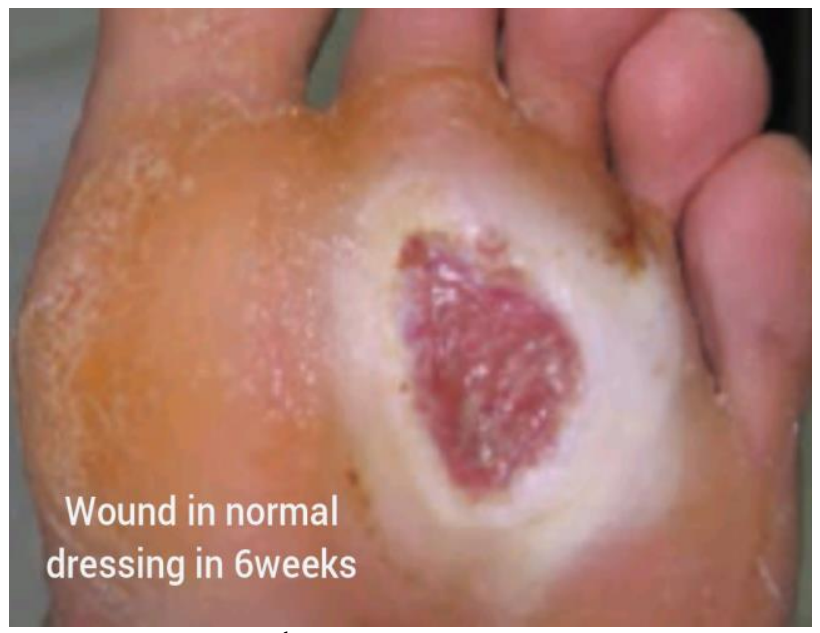

Fig. 3: Wound in $6^{\text {th }}$ week (normal dressing group)

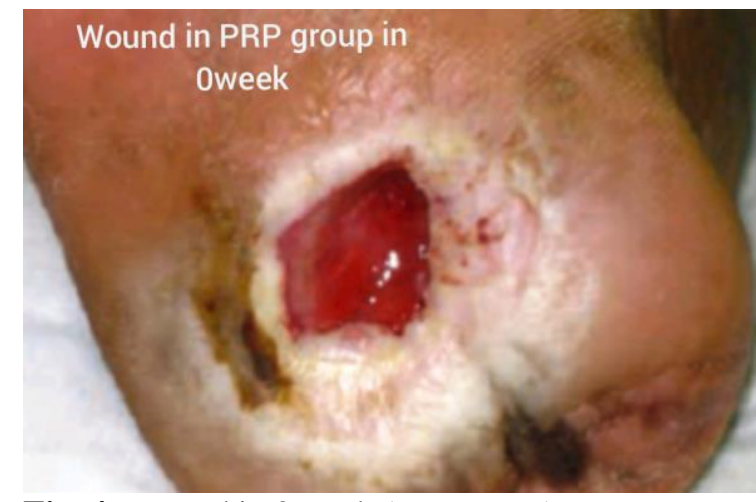

Fig. 4: Wound in 0 week (PRP group)

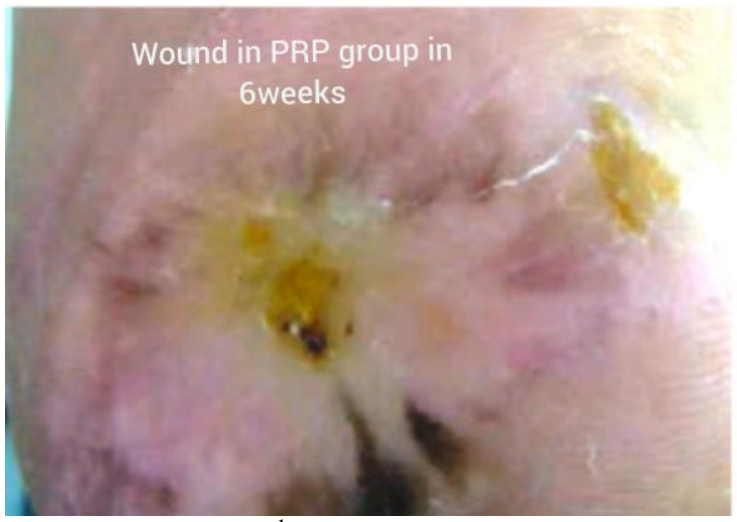

Fig. 5: Wound in $6^{\text {th }}$ week (PRP group)

\section{DISCUSSION}

Diabetic foot ulcers are chronic wounds, stuck in inflammation phase and shows cessation of epidermal growth. 120 patients of diabetic foot ulcers were studied. They were divided into two groups of 60 each. One group of patients were dressed in PRP dressings and other group with conventional dressings. A comparative study was done between both groups regarding rate of healing and fate of ulcer.

In the present study it was seen that men had increased number of diabetic foot ulcer $(68.3 \%)$ as compared to females $(31.7 \%)$. The second national data source, NHDS documented higher hospital rates in males (14). Diabetic foot ulcers are most seen in 
4th and 6th decade $(66.6 \%)$. Older the patient more chances of having diabetic foot. The prevalence of diagnosed diabetics increases with age (15) Similarly in the present study $33.3 \%$ patients were between 41 - 50 years and $33.3 \%$ were between $51-60$ years.

All patients in the present study underwent routine blood investigations including $\mathrm{HbA} 1 \mathrm{c}$ levels. Most of the patients had good glycemic control, $23 \%$ patients had HbA1c levels of $6.5-7.5(\mathrm{mmol} / \mathrm{l}), 44 \%$ patients had 7.6-8.5(mmol/l) and $33 \%$ patients had 8.6$9(\mathrm{mmol} / \mathrm{l})$. Most of the patients were on insulin for control of sugar whereas only a lesser proportion were on oral hypoglycemic agents.

All patients in the study underwent $x$-ray of foot. Patients with stress fractures and osteomyelitis were ruled out from this study. In present study, pus culture was taken for all patients and the most common organism isolated was Staphylococcus $(33 \%)$, sterile cultures were noted in $(26 \%)$ of patients and the least common organism isolated was E. coli (3\%) and Proteus (3\%). Similarly, Staphylococcus was found to be the most common organism isolated $(20 \%)$ in a study conducted in India by Banu et al., (16).

In the present study, most of the patients underwent debridement to have clean ulcer base. It has been proven by Bergstorm et al., (17) and by Goode et al., (18) that the presence of necrotic and devitalized tissue may prevent or delay wound healing. In this study, average reduction of wound area in PRP dressing compared to normal dressing was significant with a $\mathrm{P}$ value $=0.001$. Similarly, Knighton et al., (19). Conducted a prospective, randomized controlled, blinded crossover study on 32 patients. $100 \%$ epithelialization found in $81 \%$ of the PRP group and $15 \%$ of the control group by 8 weeks. After crossover all the remaining $75 \%$ in the placebo group achieved complete healing.

Systematic review was done by Villela and Santos (20) regarding the use of platelet-rich plasma for the topical treatment of chronic leg ulcers. According to the steps recommended by the Cochrane Collaboration with studies published until July 2008 this systematic review of the literature was performed. In this group 7(39\%) out of 18 selected studies, were randomized clinical trials. Among seven randomized clinical trials, five of them studied diabetic ulcers. The results showed that PRP favors the healing process $(95 \% \mathrm{Cl}: 2.94-20.31)$. The Wound Healing Society says that Platelet-derived growth factor (PDGF) is effective in treating diabetic ulcers (Level 1; Wound Healing Society 2006a).

investigated the efficiency of platelet release on the healing of chronic diabetic ulcers in comparison with platelet-poor plasma (PPP) was investigated by Setta et al. (21). In total of 24 patients, 12 patients were included in PRP group and 12 patients were included in PPP group by systematic randomization. From these results we understand the healing rate in PRP used group was faster. Overall, from this study we understand PRP dressing in diabetic ulcer is more effective in ulcer healing than normal dressing.

\section{CONCLUSION}

High concentrations of autologous GFs and cytokines into injured tissues in physiological proportions was found Platelet-Rich Plasma (PRP) treatment, which is an easy, low-cost, and minimally invasive procedure. Number of treatment modalities have been used in the treatment of chronic non-healing wounds with varying efficacy. In this present study application of platelet rich plasma into chronic non-healing diabetic foot ulcers in comparison with conventional dressing has shown a significant reduction in the size of the ulcer, hospital stay and cost for the patient. So, we suggest PRP dressing for all diabetic ulcer patients for faster and better recovery.

\section{CONFLICT OF INTEREST}

Authors declare no conflict of interest.

\section{REFERENCES}

1. Saltmarche, A. E. Low level laser therapy for healing acute and chronic wounds-the extendicare experience. International Wound Journal, 2008; 5(2): 351-360.

2. Bitsch, M., Bitsch, M., Laursen, I., Engel, A. M., Christiansen, M., Larsen, S.O., et al., Epidemiology of chronic wound patients and relation to serum levels of mannan-binding lectin. Acta Derm Venereol. 2009; 89(6): 607-611. doi: 10.2340/00015555-0730

3. Gottrup, F. Optimizing wound treatment through health care structuring and professional education. Wound Repair Regen. 2004 Mar-Apr;12(2): 129-133. doi: 10.1111/j.10671927.2004.012204. x.

4. Bennett, N. T., Schultz, G. S. Growth factors and wound healing: Part II. Role in normal and chronic wound healing. Am J Surg. 1993 Jul; 166(1): 74-81. doi: 10.1016/s00029610(05)80589-6.

5. Crovetti, G., Martinelli, G., Issi, M., Barone, M., Guizzardi, M., Campanati, B., et al., Platelet gel for healing cutaneous chronic wounds. Transfus Apher Sci. 2004 Apr; 30(2): 145151. doi: 10.1016/j.transci.2004.01.004.

6. Davis, M. D. P., Felty, C. L., Liedl, D. A., Pineda, A. A., Moore, S. B., Rooke, T. W. Autologous platelet lysate product versus placebo in patients with chronic leg ulcerations: A pilot study using a randomized, double-blind, placebo-controlled trial. Wounds, 2004; 16(9): 273-282.

7. Herouy, Y., Mellios, P., Bandemir. E., Stetter, C., Dichmann, S., Idzko, M., et al., Autologous platelet-derived wound healing factor promotes angiogenesis via alphavbeta 3 -integrin expression in chronic wounds. Int J Mol Med. 2000; Nov; 6(5): 515-519. doi: 10.3892/ijmm.6.5.515.

8. Glover, J. L., Weingarten, M. S., Buchbinder, D. S., Poucher, R. L., Deitrick, G. A., ill, Fylling. CPA 4-year outcome-based retrospective study of wound healing and limb salvage in patients with chronic wounds. Adv Wound Care. 1997; 10: 33-38.

9. Margolis, D. J., Kantor, J., Santanna, J., Strom, B. L., Berlin, J. A. Effectiveness of platelet release at the treatment of diabetic neuropathic foot ulcers. Diabetes Care. 2001; Mar; 24(3): 483-488. doi: 10.2337/diacare.24.3.483. PMID: 11289472 .

10. Lindemann, S., Tolley, N. D., Dixon, D. A., McIntyre, T. M., Prescott, S. M., Zimmerman, G. A., et al., Activated 
platelets mediate inflammatory signaling by regulated interleukin 1beta synthesis. J Cell Biol. 2001; Aug 6;154(3):485-490. doi: 10.1083/jcb.200105058. PMID:

11. Dankert, J., Krijgsveld, J., van Der Werff, J., Joldersma, W., Zaat, S. A. Platelet microbicidal activity is an important defense factor against viridans streptococcal endocarditis. J Infect Dis. 2001; 184(5): 597-605. doi: 10.1086/322802.

12. Eisenbud, D., Huang, N.F., Luke, S., Silberklang, M. Skin substitutes and wound healing: Current status and challenges. Wounds. 2004; Vol.16: 2 -17.

13. Wagner, F.W. Jr. The diabetic foot. Orthopedics. 1987; 10: 163-172.

14. Aristidis Veveshe, Giurini, J. M., Guzman, R. J. The diabetic foot-Medical and Surgical Management. 2002; 2:37.

15. Aristidis Vevese. The diabetic foot- Medical and Surgical Management edited by Aristidis Veves, 2002; 1: 18-19.

16. Banu, A., Noorul Hassan, M.M., Rajkumar, J., Srinivasa, S. Spectrum of bacteria associated with diabetic foot ulcer and biofilm formation: A prospective study. AMJ 2015; 8(9): 280-285

17. Qaseem, A., Humphrey, L. L., Forciea, M. A., Starkey, M., Denberg, T. D. Clinical Guidelines Committee of the American College of Physicians. Treatment of pressure ulcers: a clinical practice guideline from the American College of Physicians. Ann Intern Med. 2015 Mar 3; 162(5): 370-379.

18. Goode, P. S. Consensus on wound debridement. A United States perspective. Eur tissue Rep-SOC. 1995; 2: 104.

19. Knighton, D. R. 1., Ciresi, K., Fiegel, V. D., Schumerth, S., Butler, E., Cerra, F. Surg Gynecol Obstet, 1990; 170(1): 5660.

20. Villela, D. L., Santos, V. L. Evidence on the use of plateletrich plasma for diabetic ulcer: a systematic review. Growth Factors. 2010; 28(2): 111-116.

21. Saad Setta, H., Elshahat, A., Elsherbiny, K., Massoud, K., Safe, I. Platelet-rich plasma versus platelet-poor plasma in the management of chronic diabetic foot ulcers: a comparative study. Int Wound J. 2011; Jun; 8(3): 307-312. doi: 10.1111/j.1742-481X.2011.00797.x. 